\title{
Staining and peeling of the internal limiting membrane using a fluorescent dye (Rhodamine $6 \mathrm{G}$ )
}

\author{
C Haritoglou, ${ }^{1}$ T Kreutzer, ${ }^{1}$ R Tadayoni, ${ }^{2}$ H Langhals, ${ }^{3}$ C A May, ${ }^{4}$ S Thaler, ${ }^{5}$ A Kampik
}

${ }^{1}$ Department of Ophthalmology, LMU Ludwig-Maximilians-

University, Munich, Germany;

${ }^{2}$ Department of Ophthalmology, Hôpital Lariboisière, Assistance publique-Hôpitaux de Paris,

Université Paris 7, Paris, France;

${ }^{3}$ Department of Chemistry and

Biochemistry, LMU Ludwig-

Maximilians-University, Munich,

Germany; ${ }^{4}$ Department of

Anatomy, Medical Faculty "Carl

Gustav Carus," Technical

University Dresden, Dresden,

Germany; ${ }^{5}$ Centre for

Ophthalmology, University Eye

Hospital, Tübingen, Germany

Correspondence to:

Dr C Haritoglou, Department of Ophthalmology, Mathildenstr. 8, Ludwig-Maximilians-University, 80336 Munich, Germany; christos.haritoglou@med.unimuenchen.de

Accepted 16 May 2008 Published Online First

27 June 2008

\section{ABSTRACT}

Aim: To assess whether low concentrations of a fluorescent dye such as Rhodamine $6 \mathrm{G}$ would help the unaided human eye visualise the vitreous and the internal limiting membrane (ILM) under standard halogen illumination.

Material/methods: The UV/Vis absorption (E) and fluorescence (I) spectra of Rhodamine $6 \mathrm{G}$ in water were measured and compared with Indocyanine Green (ICG). Surgery was performed in two rhesus monkeys and consisted of standard pars plana vitrectomy with halogen light source used for illumination. Rhodamine $6 \mathrm{G}$ was diluted in balanced salt solution (BSS). A few drops of the dye in a concentration of $0.1 \%$ (307 m0sm) were applied over the posterior pole in the air-filled globe and washed out by irrigation after $1 \mathrm{~min}$. Immediately after surgery, the globes were enucleated, fixated and prepared for histological evaluation.

Results: In contrast to ICG, both the maximum of the absorption and emission of Rhodamin $6 \mathrm{G}$ are very much within the spectral sensitivity of the human eye. The Rhodamine 6G-BSS itself appears red in colour. Using a dye concentration of $0.1 \%$, there was no visible redstaining of the ILM as such. As the dye was irrigated out with BSS, a marked green fluorescence of the fluid within the vitreous cavity was noted. With halogen illumination through a standard 20-gauge light pipe, the dye provided a sufficient green fluorescence to identify and safely remove the ILM and to clearly differentiate areas of peeled from non-peeled ILM. During light microscopy, eyes revealed a peeled ILM demarcation with no signs of acute retinal toxicity.

Conclusion: The findings indicate that a fluorescent dye can be used for ILM peeling. Assuming that the fluorophore provides a high enough fluorescence quantum yield after adsorption to the ILM, much lower dye concentrations could be used compared with absorbent dyes, thereby minimising toxic effects.

Vital dyes help to visualise nearly transparent structures such as the internal limiting membrane (ILM) and epiretinal membranes (ERM) and therefore facilitate their removal. Indocyanine Green (ICG) was among the first dyes to be introduced for ILM staining in macular hole surgery, ${ }^{1}$ but potential toxic effects were noted shortly thereafter. ${ }^{2}$ With respect to the potential toxicity and the narrow safety margin, the applicability of ICG seems to be limited. Another dye, Trypan Blue, was introduced for epiretinal membrane staining ${ }^{4}$ and provides only a weak contrast at the level of the ILM. Brilliant Blue was suggested very recently to stain ILM as well. ${ }^{5}$ However, all dyes have in common that the contrast on the retinal surface is obtained by light absorption of the dye. Another option may be to use dye fluorescence. To further evaluate the concept of fluorescence staining, we performed a small proof-of-principle study in two primates (Rhesus monkey) using Rhodamine 6G, a dye that was shown to strongly stain the lens capsule. ${ }^{6}$ Therefore, we were confident that Rhodamine 6G would also stain the ILM, another yet thinner basement membrane. In vivo experiments performed by our group revealed dosedependent toxic effects of Rhodamine 6G. ${ }^{7}$ We also wanted to compare the light absorption (and emission) of Rhodamine 6G with the light absorption of ICG, a dye that is still in use for ILM staining.

\section{MATERIAL AND METHODS}

The UV/Vis absorption (E) of IGG as well as the UV/Vis absorption (E) and emission (I) spectra of Rhodamine 6G (Sigma-Aldrich, St. Louis, MO, RN 989-38-8, CI 45160) in water was compared with the normalised spectral sensitivity (I) of the human eye (according to CIE 1924 (8) I) and the normalised spectral characteristic of radiation of a tungsten halogen lamp (3200 K, I). The following spectrometers were used: Varian Cary 5000 for UV/Vis absorption spectra and Perkin Elmer FS 3000 Fluorescence Spectrometer totally corrected.

Two rhesus monkeys were used for this investigation. Surgery was performed in one eye; the fellow eye served as a control. The animals received veterinary care in compliance with the "Guide for the care and use of laboratory animals," and the procedures were approved by the institutional ethics committee (IMM Recherche, Paris). Ophthalmic surgery consisted of a standard pars plana vitrectomy using a standard 20 gauge system with a halogen light pipe (Alcon, Accurus). The procedure was initiated by the injection of a triamcinolone suspension (Kenacort, BristolMyers Squibb, Paris) into the vitreous cavity to allow for a reliable induction of PVD and thorough removal of the vitreous. Before the injection into the eye, the preservative benzyl alcohol-containing vehicle had been aspirated and discarded. The remaining triamcinolone crystal suspension at the bottom of the glass phial was aspirated and diluted with $0.9 \mathrm{ml}$ of balanced salt solution (BSS) (Alcon, Fort Worth, TX). Approximately $0.1 \mathrm{ml}$ of this triamcinolone suspension was then injected into the eye.

Rhodamine 6G powder was dissolved and diluted in BSS. A few drops of the dye in a concentration of $0.1 \%$ (307 $\mathrm{mOsm}$ ) were applied over the posterior pole in the air filled globe and washed out by irrigation after $1 \mathrm{~min}$. Then, the halogen light source was aimed at the retinal 


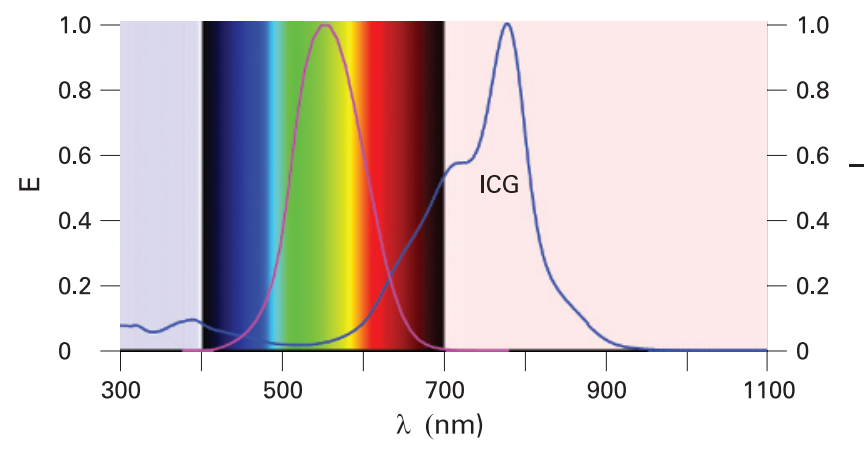

Figure 1 UV/Vis absorption (E) of Indocyanine green (ICG) (blue line) compared with the human visual spectrum (400-700 nm) (magenta line according to CIE 1924 (4a), I). The maximum absorption of ICG was at $779.0 \mathrm{~nm}$ absorption in water. Maximum sensitivity of human eye: $555 \mathrm{~nm} ; \lambda=$ wavelength.

surface. ILM peeling was performed using end-gripping forceps. Immediately after surgery, the globes were enucleated, fixated and prepared for histological evaluation. Specimens were examined using a magnification of $400 \times$.

\section{RESULTS}

The maximum of absorption of ICG is in the near infrared (NIR) at $779.0 \mathrm{~nm}$ and not within the spectral sensitivity of the human eye (maximum at $555 \mathrm{~nm}$ ) (fig 1).

For Rhodamin 6G, both the maximum of the absorption and emission are very well within the spectral sensitivity of the human eye (maximum absorption at $526.6 \mathrm{~nm}$ with a shoulder at $500 \mathrm{~nm}$ and maximum of fluorescence (emission) at $552.0 \mathrm{~nm}$ ). The small Stokes shift allows the efficient use of the radiation of tungsten halogen lamp for illumination (fig 2).

The Rhodamine 6C-BSS itself shows a red colour. Using a dye concentration of $0.2 \%$, there was no visible red-staining of the ILM as such. However, during the removal of the dye by irrigation, a marked green fluorescence of the fluid within the vitreous cavity was noted (fig 3). When aiming the halogen light source on the surface of the retina, we observed sufficient green fluorescence to identify and safely remove the ILM. The contrast between areas of peeled and unpeeled retinal surface was very pronounced (fig 4).

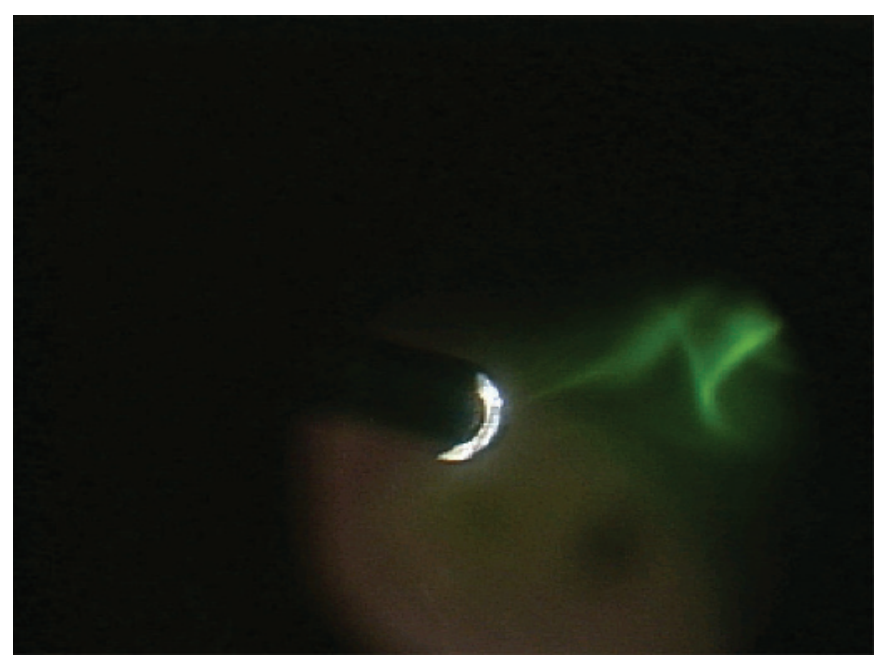

Figure 3 Visualisation of the green fluorescent dye in the vitreous cavity during irrigation.

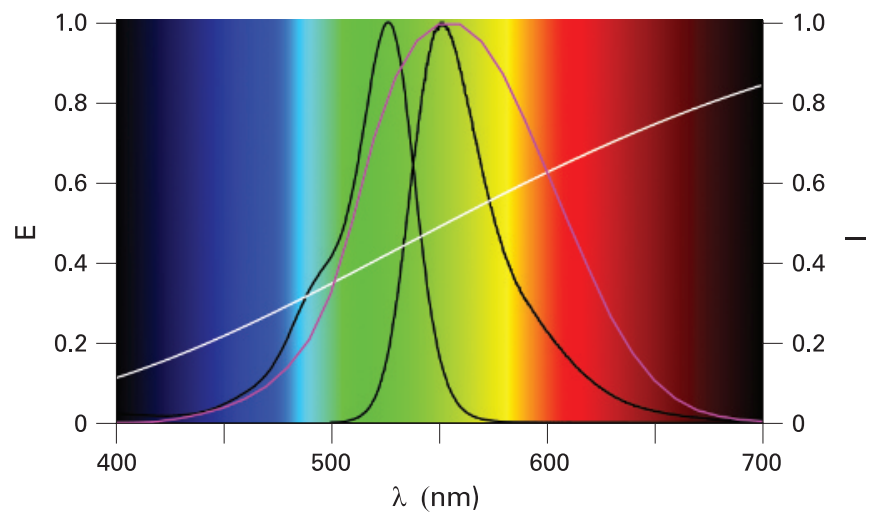

Figure 2 UV/Vis absorption (black line, left, E) and fluorescence (black line, right, I) spectra of Rhodamine $6 \mathrm{G}$ in water compared with the normalised spectral sensitivity of the human eye (magenta line according to CIE 1924 (4a), I) and the normalised spectral characteristic of radiation of a tungsten halogen lamp (white line, $3200 \mathrm{~K}$, I). For Rhodamine 6G, the maximum absorption is measured at $526.6 \mathrm{~nm}$ with a shoulder at $500 \mathrm{~nm}$ and maximum of fluorescence (emission) at $552.0 \mathrm{~nm}$; $\lambda=$ wavelength.

Light microscopy of the enucleated eyes revealed a wellpreserved ILM in most regions of the retina with no signs of acute toxic reaction. In the region of the peeled retinal surface, the ILM and some of the ganglion cell layer was partially removed. The histological appearance, however, was limited to mechanical alterations (fig 5). No toxic side effects were noted in these regions, indicating that the observed morphological alterations are likely the result of a mechanical trauma and not related to the dye itself.

\section{DISCUSSION}

ICG is not an optimal dye for staining because the majority of its absorption is in the NIR; therefore, a relatively high dye concentration for staining is required, influencing the safety margin of ICG. In contrast, the absorption and fluorescence spectra of Rhodamine $6 \mathrm{G}$ are a good compromise for visual applications because the fluorescence spectrum matches the

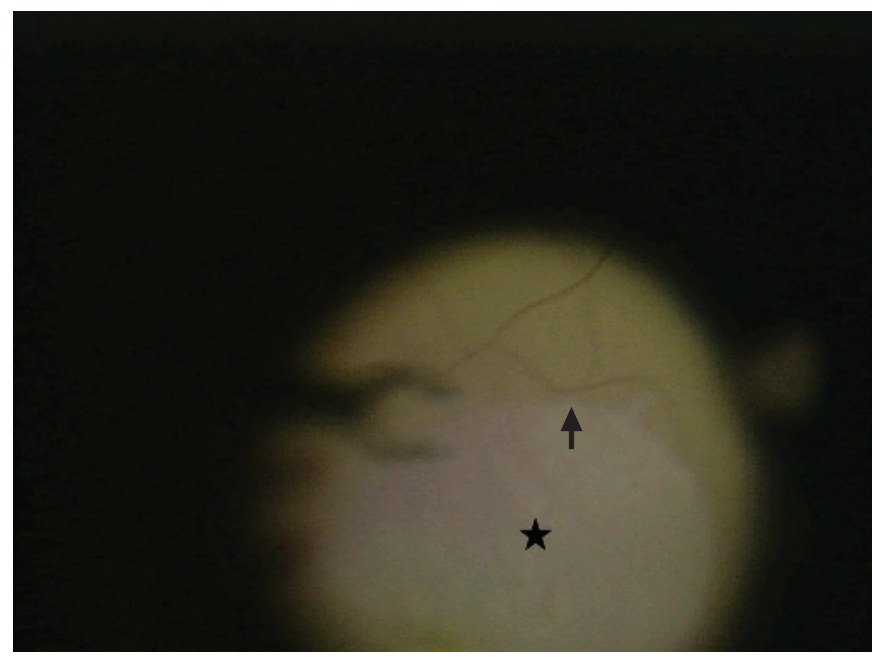

Figure 4 Peeling of the green fluorescent the internal limiting membrane (ILM) using end-gripping forceps. The area where the ILM has already been removed can be easily identified (black star). The black arrow indicates the demarcation line between peeled and unpeeled ILM. 


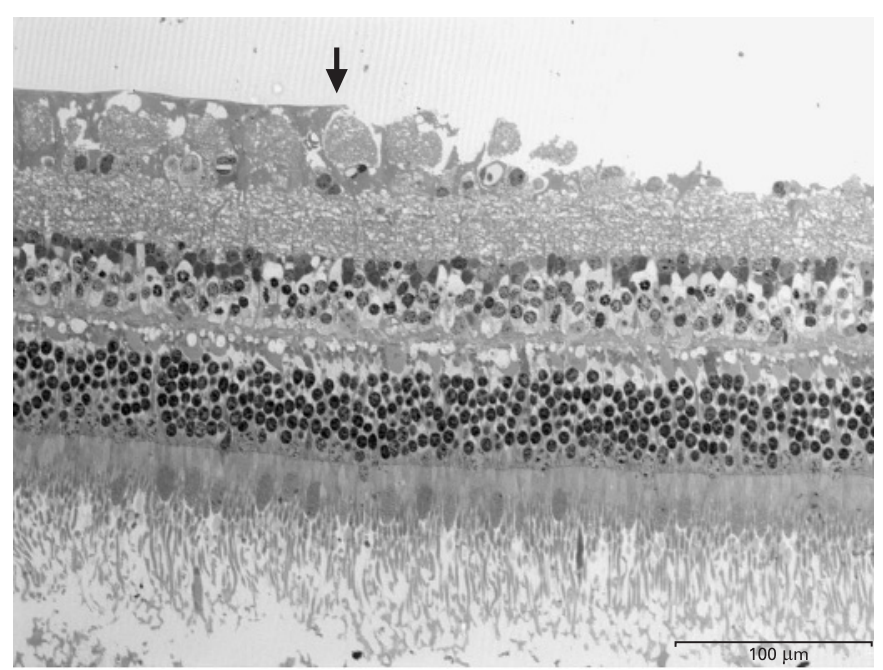

Figure 5 Light micrograph of the retina at the rim of the internal limiting membrane peeling. The semithin section was stained with Toluidine Blue. Note the normal appearance in untreated regions (left side of arrow). The peeling region shows loss of the ganglion cell layer (right side of arrow) but otherwise no tissue reaction. Specimens were examined using a magnification of $400 \times$.

spectral sensitivity of human eye quite well in the bathochromic region. In an investigation published recently, ${ }^{7}$ we evaluated the safety profile of Rhodamine $6 \mathrm{G}$ in an animal model. We found a dose-dependent toxic effect seen by a significant decrease in retinal ganglion cell (RGC) counts after intravitreal application of Rhodamine at concentrations of $0.2 \%$ and $0.5 \%$. This effect was most pronounced for concentrations of $0.5 \%$ (mean 1393 (SEM 232) cells $/ \mathrm{mm}^{2}$, as compared with BSS control group 2269 (34) cells $/ \mathrm{mm}^{2} ; \mathrm{p}<0.0001$ ). Injections with lower concentrations $(0.0002 \%, 0.002 \%$ and $0.02 \%)$ did not lead to statistically significant retinal ganglion cell loss. ERGs did not show any adverse effects, even at the highest concentrations of Rhodamine $6 \mathrm{G}$ on rods, kinetics of rhodopsin recovery after bleaching or cone-driven responses as compared with controls.

Fluorescence is an optical phenomenon, in which the molecular absorption of a photon triggers the emission of another photon with a longer wavelength. The fluorescence quantum yield describes the efficiency of the fluorescence process and is defined as the ratio of the number of photons emitted to the number of photons absorbed. Competing light is only caused by scatter and may be excluded with filters. The genuine fluorescence of human tissue becomes negligible for radiation in the visible. In theory, the fluorescence spectrum of dyes being suitable for intraocular fluorescent staining should match the sensitivity of the human eye, and the absorption should efficiently convert the light of easily accessible light sources for excitation.

Fluorescence had previously been used to visualise the vitreous. Yao and associates ${ }^{9}$ evaluated the efficacy and safety of orally administered sodium fluorescein to stain the clear vitreous before vitrectomy for proliferative diabetic retinopathy (PDR) and showed a marked staining of clear vitreous by sodium fluorescein administered $12-16$ h before surgery. In PDR patients, the staining effect seemed to be variable, and no such staining effect was seen in patients with proliferative vitreoretinopathy, indicating that this approach is only applicable in patients with breakdown of the blood-retina barrier. Although no side effects were seen in this study on 27 individuals, one drawback may still be the systemic application of fluorescein. Others injected fluorescein directly into the vitreous cavity for the same purpose. ${ }^{10}$

However, there are no reports on the use of fluorescent dyes to visualise other structures at the vitreoretinal interface. In the present proof-of-principle study, we were able to demonstrate that ILM peeling can be performed using a fluorescent dye. We conclude that the concept of ILM peeling using a fluorescent dye may point to another class of dyes being applicable for intraocular surgery. In our experiments, the green fluorescence of Rhodamine 6G was strong enough to visualise the ILM using a common halogen light source. We observed no acute dye-related toxicity in this experiment. Interestingly, there were marked alterations of the inner retinal layers in the area of peeled ILM seen in histological specimens of enucleated eyes. We do not believe that this is attributed to a toxic effect of the Rhodamine 6G itself, as adjacent areas of unpeeled ILM, which also came into contact with the dye, appeared unremarkable. We hypothesise that the observed histological alterations could be related to a mechanical trauma: as proposed for ICG ${ }^{11}$ the adsorption of Rhodamine 6G into the tissue may result in an increase in protein cross-linking and cause the ILM to be more adherent to the underlying structures, leading to ganglion layer damage as described in our present report. Large amounts of cellular debris at the retinal side of the ILM seen after ICG-assisted vitrectomy $y^{3}$ seem to underline this hypothesis. However, as such morphological alterations are not necessarily associated with unfavourable functional outcome of dye assisted vitrectomy, ${ }^{3}$ the clinical relevance of an increased rigidity of the ILM in terms of functional deficits (visual acuity, visual field, etc) remains speculative.

The application of fluorescent dyes to visualise the vitreoretinal interface implies certain advantages compared with absorbent dyes as used at present: assuming that the intraoperatively used fluorophore shows a high enough fluorescence quantum yield after having adsorbed to the ILM, with the stained structures emitting light while no light is emitted from non-stained material, much lower dye concentrations compared with absorbent dyes could theoretically be used during intraocular surgery. Using this approach, the concentration of such a fluorescent dye could be potentially reduced far beyond toxic levels.

Due to potential toxic effects of Rhodamine 6G in higher concentrations, ${ }^{67}$ we do not recommend this dye for ILM staining in humans. In addition, this study was primarily performed to assess the staining properties of the dye and immediate toxic effects, as eyes were enucleated immediately following surgery. Therefore, our approach does not represent a survival surgery, for example allowing $12-24 \mathrm{~h}$ for apoptosis to occur. Longer-term retinal toxicity had been addressed in our previous work.'

Nevertheless, Rhodamine 6G appeared to be a valuable dye for this proof-of-principle investigation. As we have seen a dosedependent toxicitity of Rhodamine 6G as mentioned above, we will now further evaluate the fluorescence of the ILM using lower concentrations of Rhodamine 6G. In addition, Rhodamine 6G will serve as a reference when investigating the safety profile and staining properties of other Rhodamine dyes as well as other known and newly synthesised fluorescent dyes with a high biocompatibility and appropriate staining characteristics. 
Acknowledgements: We thank A Horelt (Zeiss, Germany) and Zeiss (France) for the technical support, which helped us to successfully perform and document the experiments.

Competing interests: $\mathrm{CH}$ applied for a patent for the dye and the general use of fluorescent dyes for the described purpose.

Ethics approval: The procedures were approved by the institutional ethics committee (IMM Recherche, Paris).

\section{REFERENCES}

1. Kadonosono K, Itoh N, Uchio E, et al. Staining of internal limiting membrane in macular hole surgery. Arch Ophthalmol 2000;118:1116-18.

2. Kanda S, Uemura A, Yamashita T, et al. Visual field defects after intravitreous administration of indocyanine green in macular hole surgery. Arch Ophthalmol 2004;122:1447-51.

3. Haritoglou C, Gandorfer A, Gass CA, et al. Indocyanine green-assisted peeling of the internal limiting membrane in macular hole surgery affects visual outcome: a clinicopathologic correlation. Am J Ophthalmol 2002;134:836-41.
4. Feron EJ, Veckeneer M, Parys-Van Ginderdeuren R, et al. Trypan blue staining of epiretinal membranes in proliferative vitreoretinopathy. Arch Ophthalmol 2002;120:141-4.

5. Enaida $\mathbf{H}$, Hisatomi $\mathrm{T}$, Hata $\mathrm{Y}$, et al. Brilliant blue $\mathrm{G}$ selectively stains the internal limiting membrane/brilliant blue G-assisted membrane peeling. Retina 2006;26:6316.

6. Haritoglou C, Yu A, Freyer W, et al. An evaluation of novel vital dyes for intraocular surgery. Invest Ophthalmol Vis Sci 2005;46:3315-22.

7. Thaler S, Haritoglou C, Choragiewicz TJ, et al. In vivo toxicity study of Rhodamine $6 \mathrm{G}$ in the rat retina. Invest Ophthalmol Vis Sci 2008:49:2120-6.

8. Richter M. Einführung in die Farbmetrik. 2nd edn. Berlin: de Gruyter, 1981.

9. Yao Y, Wang ZJ, Wei SH, et al. Oral sodium fluorescein to improve visualization of clear vitreous during vitrectomy for proliferative diabetic retinopathy. Clin Exp Ophthalmol 2007;35:824-7.

10. Das T, Vedentham V. Intravitreal sodium fluorescein enhances visualization of clear vitreous during vitreous surgery for macular hole: a safety and efficacy study. Clin Exp Ophthalmol 2004;32:55-7.

11. Wollensak G, Spoerl E, Wirbelauer C, et al. Influence of indocyanine green staining on the biomechanical strength of porcine internal limiting membrane. Ophthalmologica 2004;218:278-82.

\section{BMJ Masterclasses}

BMJ Masterclasses are educational meetings designed specifically to meet the learning needs of doctors. They help doctors keep up to date with the latest evidence and recent guidelines in major clinical areas, enabling them to use the latest evidence to make better decisions. The latest evidence, recent guidelines and best practice are delivered in an interactive and informative manner by leading experts. The speakers are specifically chosen as highly-skilled communicators who can authoritatively enthuse the audience and interpret the latest research and guidelines into practical tips for busy doctors. BMJ Masterclasses have proved a huge hit with clinicians, with many saying they have influenced their clinical practice.

http://masterclasses.bmj.com/

$\mathrm{BMJ}$

\section{masterclasses}




\title{
BJO
}

\section{Staining and peeling of the internal limiting membrane using a fluorescent dye (Rhodamine $6 \mathrm{G}$ )}

\author{
C Haritoglou, T Kreutzer, R Tadayoni, et al.
}

Br J Ophthalmol 2008 92: 1265-1268 originally published online June 27, 2008

doi: 10.1136/bjo.2008.141911

Updated information and services can be found at:

http://bjo.bmj.com/content/92/9/1265.full.html

\section{These include:}

References This article cites 10 articles, 2 of which can be accessed free at: http://bjo.bmj.com/content/92/9/1265.full.html\#ref-list-1

Email alerting Receive free email alerts when new articles cite this article. Sign up in service the box at the top right corner of the online article.

Topic Articles on similar topics can be found in the following collections Collections

Notes

To request permissions go to:

http://group.bmj.com/group/rights-licensing/permissions

To order reprints go to:

http://journals.bmj.com/cgi/reprintform

To subscribe to BMJ go to:

http://group.bmj.com/subscribe/ 